\title{
CHAINS AND ANTICHAINS IN PARTIAL ORDERINGS
}

\author{
VALENTINA S. HARIZANOV, CARL G. JOCKUSCH, JR., AND JULIA F. KNIGHT
}

\begin{abstract}
We study the complexity of infinite chains and antichains in computable partial orderings. We show that there is a computable partial ordering which has an infinite chain but none that is $\Sigma_{1}^{1}$ or $\Pi_{1}^{1}$, and also obtain the analogous result for antichains. On the other hand, we show that every computable partial ordering which has an infinite chain must have an infinite chain that is the difference of two $\Pi_{1}^{1}$ sets. Our main result is that there is a computably axiomatizable theory $K$ of partial orderings such that $K$ has a computable model with arbitrarily long finite chains but no computable model with an infinite chain. We also prove the corresponding result for antichains. Finally, we prove that if a computable partial ordering $\mathcal{A}$ has the feature that for every $\mathcal{B} \cong \mathcal{A}$, there is an infinite chain or antichain that is $\Delta_{2}^{0}$ relative to $\mathcal{B}$, then we have uniform dichotomy: either for all copies $\mathcal{B}$ of $\mathcal{A}$, there is an infinite chain that is $\Delta_{2}^{0}$ relative to $\mathcal{B}$, or for all copies $\mathcal{B}$ of $\mathcal{A}$, there is an infinite antichain that is $\Delta_{2}^{0}$ relative to $\mathcal{B}$.
\end{abstract}

A partial ordering is a pair $(P,<)$, where $P$ is a nonempty set and $<$ is a binary relation on $P$ which is transitive and irreflexive. A subset $C \subseteq P$ is a chain if $(C,<)$ is linearly ordered. A subset $A \subseteq P$ is an antichain if no two distinct elements of $A$ are comparable under $<$. It follows from Ramsey's theorem for pairs that every infinite partial ordering has an infinite chain or an infinite antichain.

It follows from an effective version of Ramsey's theorem for pairs, due to Jockusch (Remark on page 273 in [6]), that a computable partial ordering of $\omega$ has either an infinite $\Delta_{2}^{0}$ chain, or an infinite $\Delta_{2}^{0}$ antichain, or else both an infinite $\Pi_{2}^{0}$ chain and an infinite $\Pi_{2}^{0}$ antichain. On the other hand, Herrmann [5] showed that there is a computable partial ordering of $\omega$ with no infinite $\Sigma_{2}^{0}$ chain or antichain. In [3], there are some interesting related results on trees. If we restrict attention to infinite chains (or infinite antichains), assuming they exist in a given computable partial ordering, the complexity bounds are much higher.

We show that there is a computable partial ordering with an infinite chain but none that is $\Sigma_{1}^{1}$ or $\Pi_{1}^{1}$, and we obtain the analogous result for antichains. On the other hand, the Kleene Basis Theorem implies that every computable partial ordering with an infinite chain (or antichain) has one that is Turing reducible to Kleene's $\mathcal{O}$ (and thus to a $\Pi_{1}^{1}$ set). For chains, we can improve this result to show that every computable partial ordering with an infinite chain has one that is $d-\Pi_{1}^{1}$, i.e., the difference of two $\Pi_{1}^{1}$ sets. The analogous question for antichains is open, but a straightforward modification of the Kleene Basis Theorem shows that every computable partial ordering with an infinite antichain has one that is truth-table reducible to Kleene's $\mathcal{O}$.

Acknowledgments. Harizanov was partially supported by the NSF grant DMS-0704256. Jockusch was supported by NSFC Grand International Joint Project "New Directions in Theory and Applications of Models of Computation," No. 60310213. 
By the Compactness Theorem, if $K$ is a theory of partial orderings, and there are models with arbitrarily large finite chains (or antichains), then there is a model with an infinite chain (or antichain). It is natural to ask the following questions for a computably axiomatizable theory $K$.

Question 0.1. If $K$ has a computable model with arbitrarily large finite chains, must $K$ have a computable model with an infinite chain?

Question 0.2. If $K$ has a computable model with arbitrarily large finite antichains, must $K$ have a computable model with an infinite antichain?

We answer both questions negatively. In contrast, we give an example of a theory $K$, not computably axiomatizable, such that $K$ has computable models, some (noncomputable) model of $K$ has arbitrarily large finite chains but no infinite chain, and all computable models have infinite chains. We give a similar example for antichains.

We consider partial orderings $\mathcal{A}$ such that for all copies $\mathcal{B}$ of $\mathcal{A}$ there is a chain or antichain that is $\Delta_{2}^{0}$ relative to $\mathcal{B}$. We obtain a uniform dichotomy result in this setting; i.e., either we always get a chain, or else we always get an antichain.

Section 1 gives results on the definability of infinite chains and antichains in computable partial orderings, as well as some results on the complexity of determining whether a partial ordering has an infinite chain, or an infinite antichain. Trees play a crucial role in this section. The examples showing the failure of computable compactness for chains and antichains are given in Section 2, with some related results. The uniformity result for chains in copies of a partial ordering is in Section 3. Section 4 gives some open problems.

\section{Complexity BOUnds fOR Chains AlONe, AND FOR ANTICHAins AlONE}

We consider what can be said about definability properties of infinite chains (antichains) in computable partial orderings which have them. This section uses heavily the work and ideas of S. C. Kleene. In a computable partial ordering, the infinite chains form a $\Pi_{2}^{0}$ subset of $2^{\omega}$. Thus, by the Kleene Basis Theorem ([7], page 420), every computable partial ordering with an infinite chain must have an infinite chain that is Turing reducible to some $\Pi_{1}^{1}$ set. Analogous remarks hold for antichains. The following theorems show that these results are best possible with respect to the analytical hierarchy.

Theorem 1.1. There is a computable partial ordering with an infinite chain, but not one that is $\Sigma_{1}^{1}$ or $\Pi_{1}^{1}$.

Proof. Kleene produced a computable tree $T \subseteq \omega^{<\omega}$ such that $T$ has infinite paths, but no infinite hyperarithmetical paths. Let $T$ be partially ordered by the extension relation. Then every path in $T$ is an infinite chain in this partial ordering (so the partial ordering has infinite chains). Every infinite chain computes a path through $T$; namely, its downward closure, where this is obtained by closing under initial segments. Hence, there are no infinite hyperarithmetical chains. Suppose now, for the sake of obtaining a contradiction, that there were an infinite $\Sigma_{1}^{1}$ chain $C$. Let $D$ be the downward closure of $C$, i.e.,

$$
D=\left\{\sigma \in \omega^{<\omega}:(\exists \tau)[\tau \in C \& \sigma \subseteq \tau]\right\} .
$$


Then $D$ is a path through $T$, so $D$ is not hyperarithmetical. Since $C$ is $\Sigma_{1}^{1}$, the path $D$ is also $\Sigma_{1}^{1}$. Since $D$ contains exactly one string of each length, the following holds for all $\sigma \in \omega^{<\omega}$ :

$$
\sigma \notin D \Longleftrightarrow(\exists \tau)[\tau \in D \&|\tau|=|\sigma| \& \tau \neq \sigma] .
$$

It follows that the complement of $D$ is also $\Sigma_{1}^{1}$, so $D$ is $\Pi_{1}^{1}$. Then $D$ is hyperarithmetical, which is a contradiction. A similar contradiction arises if we assume that the partial ordering of $T$ by inclusion has an infinite $\Pi_{1}^{1}$ chain (or we may use the fact that every infinite $\Pi_{1}^{1}$ set has an infinite hyperarithmetical subset, see [7], page 404).

We remark that the partial ordering above has an infinite chain that is the difference of two $\Pi_{1}^{1}$ sets, namely, the leftmost path through $T$. It is natural to ask whether in every computable partial ordering which has an infinite chain, there is an infinite chain that is the difference of two $\Pi_{1}^{1}$ sets.

Theorem 1.2. Let $\mathcal{A}$ be a computable partial ordering. If there is an infinite chain, then there is one that is $d-\Pi_{1}^{1}$.

Proof. We let $\mathcal{A}=(A,<)$, where $A \subseteq \omega$. We shall use both the partial ordering < and the usual ordering on $\omega$, which we denote by $<_{\omega}$. By a result of Tennenbaum, the given chain in $\mathcal{A}$ has a subset of order type $\omega$ or $\omega^{*}$. (To prove this, apply Ramsey's Theorem for pairs to the result of coloring a pair red if its elements are ordered in the same way by $<$ and $<_{\omega}$, and blue otherwise.) If there is a chain in $\mathcal{A}$ of order type $\omega$, then there is a function $f$ on $\omega$ such that for all $n, f(n)<f(n+1)$ and $f(n)<_{\omega} f(n+1)$. Similarly, if there is a chain in $\mathcal{A}$ of order type $\omega^{*}$, then there is a function $f$ on $\omega$ such that for all $n, f(n)>f(n+1)$ and $f(n)<_{\omega} f(n+1)$. Without loss of generality, we suppose that there is a function $f$ of the first kind, strictly increasing in both $<$ and $<_{\omega}$. Let $S$ be the set of all $a \in \mathcal{A}$ such that there exists such a function $f$ with $a \in \operatorname{ran}(f)$. Then $S$ is $\Sigma_{1}^{1}$. Note that $S$ is an initial segment of $\mathcal{A}$, in the sense that if $a \in S$ and $b<a$, then $b \in S$.

Our infinite $d-\Pi_{1}^{1}$ chain $C$ will be the range of the function $f$, where $f(0)$ is the $<_{\omega}$-least element of $S$, and for each $n, f(n+1)$ is the $<_{\omega}$-least $a \in S$ such that $a>f(n)$ and $a>_{\omega} f(n)$. Note that $f$ is total and $1-1$, so the range $C$ of $f$ is infinite. To show that $C$ is $d$ - $\Pi_{1}^{1}$, it suffices to produce a $\Pi_{1}^{1}$ set $P$ such that $C=P-\bar{S}=P \cap S$. To this end, we first define a $\Pi_{1}^{1}$ set $A \subseteq \omega^{<\omega}$. The elements of $A$ may be thought of as "candidates" for initial segments of $f$. In fact, every nonempty finite initial segment of $f$ will be in $A$, but the converse need not hold. Specifically, let $A$ be the set of strings $\sigma \in \omega^{<\omega}$ such that the following three conditions hold:

(1) $\sigma(0)=f(0)$;

(2) $\sigma$ is strictly increasing in both $<$ and $<_{\omega}$;

(3) For all $i$ and $x$, if $\sigma(i+1)$ is defined and $\sigma(i)<_{\omega} x<_{\omega} \sigma(i+1)$ and $\sigma(i)<x$, then $x \notin S$.

The set $A$ is $\Pi_{1}^{1}$ because $S$ is $\Sigma_{1}^{1}$ and $<$ is computable. It is easy to check that every nonempty finite initial segment of $f$ is in $A$. Let $P$ be the union of the sets $\operatorname{ran}(\sigma)$ for $\sigma \in A$. The set $P$ is $\Pi_{1}^{1}$ because $A$ is $\Pi_{1}^{1}$. Also, $C \subseteq P$ because $C=\operatorname{ran}(f)$ and every nonempty initial segment of $f$ is in $A$. Because $C \subseteq S$ by the definition of $f$, it follows that $C \subseteq P \cap S$. 
It remains to show that $P \cap S \subseteq C$. Let $n \in P \cap S$. Since $n \in P$, we may choose $\sigma \in A$ with $n$ in $\operatorname{ran}(\sigma)$. Say $n=\sigma(k)$. Let $\tau=\sigma \uparrow(k+1)$, so $\sigma$ extends $\tau$, and $n$ is the final value of $\tau$. Note that $\tau \in A$. Since $\tau$ is increasing in the ordering < and $n$ is its final value, $n$ is the <-greatest value of its range. As $n \in S$ and $S$ is an initial segment of $<\operatorname{ran}(\tau) \subseteq S$. It is now easy to check, by induction on $i$, that $\tau(i)=f(i)$ for all $i<|\tau|=k+1$. In particular, $n=\sigma(k)=\tau(k) \in \operatorname{ran}(f)=C$, as needed to complete the proof that $P \cap S \subseteq C$. Hence, $C=P \cap S$ is $d$ - $\Pi_{1}^{1}$.

We do not know whether every computable partial ordering with an infinite antichain has one that is $d$ - $\Pi_{1}^{1}$, but it does not seem possible to obtain such a result by the method of the previous theorem. The next remark gives a weaker form of this result, though.

Remark 1.3. Suppose $\mathcal{A}$ is a computable partial ordering with an infinite antichain. Then $\mathcal{A}$ has an infinite antichain that is truth-table reducible to Kleene's $\mathcal{O}$.

Proof. We first prove a basis result for $\Pi_{1}^{0}$ sets which is a variant of the Kleene Basis Theorem. Suppose $P$ is a $\Pi_{1}^{0}$ subset of $2^{\omega}$ which has an infinite element (i.e., infinite as a subset of $\omega$ ). Then $P$ has an infinite element $A$ that is truth-table reducible to $\mathcal{O}$. To prove this, let $Q$ be the set of strictly increasing functions $g$ whose range is in $P$. Note that $Q$ is a nonempty $\Pi_{1}^{0}$ class, and so has a lexicographically least element $f$. Let $I$ be the set of strings that are initial segments of $f$, and let $E$ be the set of strings that are finite initial segments of functions in $Q$. Then $E$ is $\Sigma_{1}^{1}$. Note that for all increasing $\sigma \in \omega^{<\omega}$ and all $i \in \omega$,

$$
\sigma^{\wedge} i \in I \Longleftrightarrow\left(\sigma \in I \& \sigma^{\wedge} i \in E \&(\forall j<i)\left[\sigma^{\wedge} j \notin E\right]\right) \text {. }
$$

Using the fact that $E$ is $\Sigma_{1}^{1}$, we easily see that $I$ is $d-\Pi_{1}^{1}$. Let $A=\operatorname{ran}(f)$. It follows that $A$ is truth-table reducible to $\mathcal{O}$, since for all $n, n \in A$ iff $n \in \operatorname{ran}(\sigma)$ for some $\sigma \in(n+1)^{n+1} \cap I$. Applying this to the set $P$ of antichains of a given computable partial ordering, we see that every computable partial ordering with an infinite antichain has an infinite antichain that is truth-table reducible to $\mathcal{O}$.

We don't know whether the above remark can be improved to show that every computable partial ordering with an infinite antichain has an infinite antichain that is bounded truth-table reducible to $\mathcal{O}$. If this were the case, it would follow that every computable partial ordering which has an infinite antichain has one that is $d$ - $\Pi_{1}^{1}$. This is because the sets that are bounded truth-table reducible to $\mathcal{O}$ coincide with the finite Boolean combinations of $\Pi_{1}^{1}$ sets, which in turn coincide with the finite unions of $d-\Pi_{1}^{1}$ sets.

Theorem 1.4. There is a computable partial ordering with an infinite antichain, but not one that is $\Sigma_{1}^{1}$ or $\Pi_{1}^{1}$.

Proof. We define an effective transformation $\Phi$ of trees into partial orderings such that $T$ has a path iff $\Phi(T)$ has an infinite antichain. We let $\Phi(T)=(T,<)$, where $\sigma<\tau$ if for the greatest common ancestor $\rho$, we have $\rho^{\wedge} i \subseteq \sigma$ and $\rho^{\wedge} j \subseteq \tau$ where $i<j$. If one of $\sigma, \tau$ is an initial segment of the other, then $\sigma$ and $\tau$ are not comparable under $<$. We must show that $<$ is a partial ordering on $T$. Clearly, $\sigma \nless \sigma$. Suppose $\sigma<\nu<\tau$. Let $\rho$ be the greatest common ancestor of $\sigma$ and $\nu$, and let $\rho^{\prime}$ be the greatest common ancestor of $\nu$ and $\tau$. Recall that $|\rho|$ denotes the length of $\rho$. There are three cases to consider: $\left|\rho^{\prime}\right|<|\rho|,|\rho|<\left|\rho^{\prime}\right|$, and $|\rho|=\left|\rho^{\prime}\right|$ (which means that $\rho=\rho^{\prime}$ ). In all three cases, we can see that $\sigma<\tau$. Therefore, 
$<$ is a strict partial ordering. We can see that $\sigma$ and $\tau$ are comparable under $<$ iff neither is an extension of the other. Thus, the antichains in $T$ under the ordering $<$ defined above coincide with the chains in $T$ under the extension ordering. By the proof of Theorem 1.1, there are infinite chains in $T$ under the extension ordering, but none that are $\Sigma_{1}^{1}$ or $\Pi_{1}^{1}$.

\section{Failure of Computable compactness}

It is an easy consequence of the usual Compactness Theorem that every theory of partial orderings which for each $n \in \omega$ has a model with a chain of length $n$ must have a model with an infinite chain. The following theorem implies that the analogous result for computable chains in computable partial orderings does not hold.

Theorem 2.1. There is a computably axiomatizable theory $K$ of partial orderings such that:

(1) $K$ has a computable model $\mathcal{C}$ with arbitrarily long finite chains, and

(2) no computable model of $K$ has an infinite chain.

Proof. Before giving the axioms for $K$, we will define the computable partial ordering $\mathcal{C}$ used for (1). The axioms for $K$ will then be a partial description of $\mathcal{C}$. Let $\left\{A_{n}\right\}_{n \in \omega}$ be a computable sequence of finite partial orderings satisfying the following conditions for all $m, n \in \omega$.

(i) If $m \neq n$, then $A_{m}$ cannot be embedded in $A_{n}$.

(ii) $A_{n}$ has no chain of length greater than 3 .

(iii) Let $G_{n}$ be the undirected graph whose vertices are the points of $A_{n}$ and whose edges join comparable distinct points in $A_{n}$. Then for any distinct vertices $v, w$ of $G_{n}$, there is a unique path from $v$ to $w$.

The existence of such a sequence $\left\{A_{n}\right\}_{n \in \omega}$ has long been known. In particular, we choose $A_{n}$ as follows. The ordering $A_{n}$ has $2 n+7$ distinct elements $b_{0}, b_{1}, \ldots, b_{n+1}, m_{0}, m_{1}, \ldots, m_{n+2}, t_{0}, t_{1}$. These elements are ordered as shown in Figure 1. It is clear that $A_{n}$ has properties (i)-(iii).

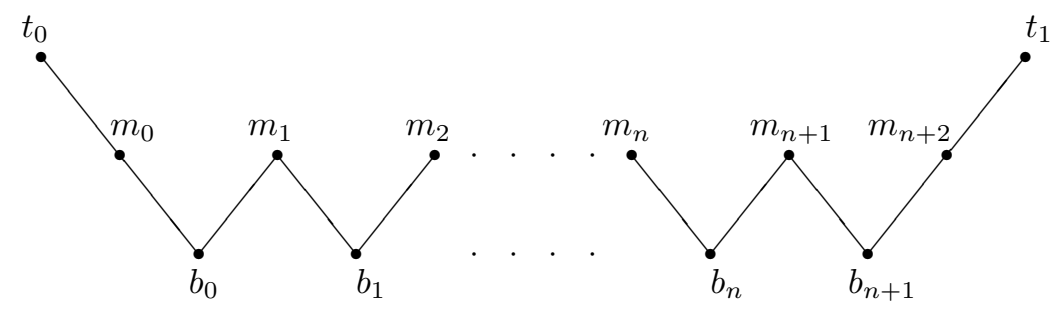

Figure 1. $A_{n}$

We use the letters $\sigma, \tau$, and $\mu$ to denote elements of $2^{<\omega}$. We identify a binary string with its Gödel number, and, in particular, $A_{\sigma}$ denotes $A_{n}$, where $n$ is the Gödel number of the string $\sigma$. For $\sigma \in 2^{<\omega}$, let the partial ordering $C_{\sigma}$ be defined as follows: $C_{\sigma}$ has a special chain $a_{0}, a_{1}, \ldots, a_{s}$ with $a_{0}<a_{1}<\cdots<a_{s}$, where 
$s=|\sigma|$, and $|\sigma|$ denotes the length of $\sigma$. For each $i<s$, there is a copy of $A_{\sigma \nmid i}$ in $C_{\sigma}$. This copy is attached so that the element that plays the role of $t_{0}$ is below $a_{i}$. Thus, the elements that play the roles of $t_{0}, m_{0}$, and $b_{0}$ are below $a_{i}$, but all other elements of the copy are incomparable with all elements of $C_{\sigma}$ not within the copy. Of course, pairwise disjoint copies are used as $i$ varies, and also the copies are disjoint from the special chain. See Figure 2.

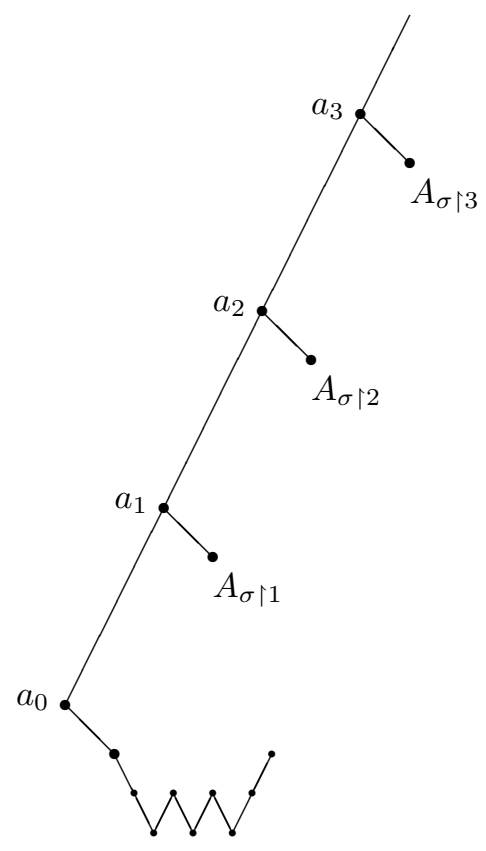

Figure 2. $C_{\sigma}$, with $A_{\sigma\lceil 0}$ indicated

Let $T$ be an infinite computable tree of binary strings with no infinite computable path. Let the partial ordering $\mathcal{C}$ be the disjoint union of the partial orderings $C_{\sigma}$ for $\sigma \in T$. If $\sigma$ and $\tau$ are distinct elements of $T$, then all elements of $C_{\sigma}$ are incomparable with all elements of $C_{\tau}$ in the partial ordering $\mathcal{C}$. The partial ordering $\mathcal{C}$ has arbitrarily long chains since $T$ is infinite, and $\mathcal{C}$ can clearly be made computable.

In any partial ordering, for every $\sigma \in 2^{<\omega}$, let $B_{\sigma}$ be the set of elements $x$ of the partial ordering such that there exist $t<x$ and an embedding $p$ of $A_{\sigma}$ into the partial ordering with $p\left(t_{0}\right)=t$. Note that $B_{\sigma}$ is definable by an elementary first-order formula, independent of the partial ordering; moreover, the formula can be effectively computed from $\sigma$. We are now ready to give the axioms for $K$.

A1. First, there are the axioms for partial orderings.

A2. Next, for each $\sigma \notin T$, there is an axiom saying that $B_{\sigma}$ is empty. 
A3. For each $\sigma$ in $T$, there is an axiom saying

$$
(\forall x)(\forall y)\left[\left(x<y \wedge x \in B_{\sigma}\right) \Longrightarrow\left(y \in B_{\sigma^{\wedge} 0} \vee y \in B_{\sigma^{\wedge} 1}\right)\right]
$$

A4. For all incompatible $\sigma, \tau$, there is an axiom asserting that $B_{\sigma} \cap B_{\tau}$ is empty.

A5. There is an axiom asserting that for any four distinct comparable elements, the greatest of them is in $B_{\emptyset}$.

A6. There is an axiom saying that for each $x \in B_{\emptyset}$, there is a least $y \leq x$ such that $y \in B_{\emptyset}$.

A7. Finally, there is an axiom saying that for each $x \in B_{\emptyset}$, then there do not exist $y, z>x$ such that $y, z$ are incomparable.

The following lemma says that the only elements of $B_{\sigma}$ in $\mathcal{C}$ are the obvious ones. This will make it easy to show that $\mathcal{C}$ is a model of $K$.

Lemma 2.2. In $\mathcal{C}$, the set $B_{\sigma}$ consists of all $x$ such that there exists a string $\tau$ extending $\sigma$ with $\tau \in T$, and $x=a_{j}$ in $C_{\tau}$ for some $j$ with $|\tau| \geq j \geq|\sigma|$.

Proof. If $x$ is as described above, then clearly, $x \in B_{\sigma}$. Suppose now that $x \in B_{\sigma}$. Let $F$ be a subordering of $\mathcal{C}$ such that $F$ is isomorphic to $A_{\sigma}$ and $x>_{C} t_{0}^{F}$. Here we fix an isomorphism from $A_{\sigma}$ to $F$ and denote the image of $a$ by $a^{F}$ for $a \in A_{\sigma}$. Let $\tau$ be the unique binary string such that $x \in C_{\tau}$. Clearly, $\tau \in T$.

We write the special chain for $C_{\tau}$ as $a_{0}, a_{1}, \ldots, a_{|\tau|}$, and we write $d^{i}$ for the image of $d$ under a fixed isomorphism from $A_{\tau \uparrow i}$ to the copy of this ordering having an element below $a_{i}$. Since $x$ properly bounds a chain of length 3 in $C_{\tau}, x$ belongs to the special chain of $C_{\tau}$. Let $x$ be the element $a_{j}$ of the special chain of $C_{\tau}$. The element $b_{0}^{F}$ satisfies $b_{0}^{F}<_{C} t_{0}^{F}<_{C} x=a_{j}$, so $b_{0}^{F}$ lies below some element of the special chain. Furthermore, $b_{0}^{F}$ has two incomparable elements above it, namely $m_{0}^{F}$ and $m_{1}^{F}$. Every element of $C_{\tau}$ that lies below some element of the special chain and has two incomparable elements above it has the form $b_{0}^{i}$ for some $i$. Fix $i$ such that $b_{0}^{F}=b_{0}^{i}$. Note that $a_{i}$ is the least element of the special chain that is above $b_{0}^{i}$ and $x=a_{j}>_{C} b_{0}^{i}$, so $a_{j} \geq_{C} a_{i}$, and hence $j \geq i$.

We claim that $F$ is embeddable in $A_{\tau \uparrow i}$. Since $F$ is isomorphic to $A_{\sigma}$, it follows from the claim that $A_{\sigma}$ is also embeddable in $A_{\tau \uparrow i}$. Since the $A_{k}$ 's are pairwise non-embeddable, it follows from the claim that $\sigma=\tau \uparrow i$, so $\tau$ extends $\sigma$. Since $|\tau| \geq j \geq i$, the lemma follows from the claim.

To prove the claim, we first show that

$$
F-\left\{m_{0}^{F}, t_{0}^{F}\right\} \subseteq A_{\tau \uparrow i}-\left\{m_{0}^{i}, t_{0}^{i}\right\} .
$$

Let $H=A_{\tau \uparrow i}-\left\{m_{0}^{i}, t_{0}^{i}\right\}$. Observe that if $y \in H-\left\{b_{0}^{i}\right\}$ and $z$ is comparable with $y$, then $z \in H$. List $F-\left\{m_{0}^{F}, t_{0}^{F}\right\}$ without repetitions as $b_{0}^{F}, m_{1}^{F}, b_{1}^{F}, \ldots$, where each element of this list except the first one is comparable with the previous one. Since $b_{0}^{F}=b_{0}^{i}$, we obtain that $b_{0}^{F} \in H$. We have noted that $b_{0}^{F}=b_{0}^{i}$. Note that $m_{1}^{i}$ is above $b_{0}^{i}$, but is not below any element of the special chain, and, furthermore, it is the unique element with this property. Now, consider $m_{1}^{F}$. It is also above $b_{0}^{i}$, since $b_{0}^{i}=b_{0}^{F}$. Note that no two incomparable elements of $C_{\tau}$ having a common lower bound both lie below elements of the special chain. Clearly, $t_{0}^{F}$ and $m_{1}^{F}$ 
are incomparable and have $b_{0}^{F}$ as a common lower bound. Also $t_{0}^{F}$ lies below the element $x$ of the special chain. Thus, $m_{1}^{F}$ does not lie below any element of the special chain. Since $m_{1}^{i}$ and $m_{1}^{F}$ are both above $b_{0}^{i}$, but not below any element of the special chain, we have that $m_{1}^{i}=m_{1}^{F}$. It follows that $m_{1}^{F} \in H$. It now follows by induction on the list (starting with $m_{1}^{F}$ ) that every element of the list is in $H$, using the fact that every element except the first one is comparable with the previous element. Thus, $F-\left\{m_{0}^{F}, t_{0}^{F}\right\} \subseteq A_{\tau \uparrow i}-\left\{m_{0}^{i}, t_{0}^{i}\right\}$. We now define an embedding $q$ of $F$ into $A_{\tau \uparrow i}$. Let $q(x)=x$ for $x \in F-\left\{m_{0}^{F}, t_{0}^{F}\right\}$, and let $q\left(m_{0}^{F}\right)=m_{0}^{i}$ and $q\left(t_{0}^{F}\right)=t_{0}^{i}$. It is easy to check that this is an embedding, using the fact that $F-\left\{m_{0}^{F}, t_{0}^{F}\right\} \subseteq A_{\tau \uparrow i}-\left\{m_{0}^{i}, t_{0}^{i}\right\}$.

Lemma 2.3. $\mathcal{C}$ is a model of $K$.

Proof. This lemma is a straightforward consequence of Lemma 2.2. We check that $\mathcal{C}$ satisfies A3 and leave verification of the other axioms to the reader. To verify A3, fix $\sigma \in T$ and $x, y \in C$ such that $x<_{C} y$ and $x \in B_{\sigma}$. By Lemma 2.2, we may fix a string $\tau$ extending $\sigma$ with $\tau \in T$, and $x=a_{j}$ in $C_{\tau}$ for some $j$ with $|\tau| \geq j \geq|\sigma|$. Since $x<_{C} y$, we have that $y=a_{k}$ in $C_{\tau}$ for some $k$ with $|\tau| \geq k>j$. Take $i \leq 1$ such that $\tau$ extends $\sigma^{\wedge} i$. Such an $i$ exists because $|\tau|>j \geq \sigma$. Then $y \in B_{\sigma^{\wedge} i}$ via the witnesses $\tau$ and $k$, by the easy direction of Lemma 2.2.

Now, let $\mathcal{A}$ be a computable model of $K$. Suppose that $\mathcal{A}$ has an infinite chain $R_{0}$. We shall obtain a contradiction by first showing that $\mathcal{A}$ has an infinite computable chain $R$ and then using $R$ to obtain a computable path through $T$. It follows from A5 that $R_{0}$ contains at most three elements not in $B_{\emptyset}$. By discarding these elements we may assume that $R_{0} \subseteq B_{\emptyset}$. Thus, by $\mathrm{A} 6$, for each $x \in R_{0}$ there is a least $y \leq x$ such that $y \in B_{\emptyset}$. This least $y$ is denoted $\mu(x)$. Note that $\mu$ is defined on all elements of $R_{0}$.

Lemma 2.4. $\mu$ is constant on $R_{0}$.

Proof. Suppose that $a, b \in R_{0}$. We must show that $\mu(a)=\mu(b)$. We assume without loss of generality that $a \leq b$ (since $R_{0}$ is a chain). Hence $\mu(a) \leq a \leq b$ and $\mu(a) \in B_{\emptyset}$. It follows from the definition of $\mu(b)$ that $\mu(b) \leq \mu(a)$. Therefore, $\mu(b) \leq \mu(a) \leq a$ and $\mu(b) \in B_{\emptyset}$, so it follows from the definition of $\mu(a)$ that $\mu(a) \leq \mu(b)$. Because $\mu(a) \leq \mu(b)$ and $\mu(b) \leq \mu(a)$, we conclude that $\mu(a)=\mu(b)$, as required.

Lemma 2.5. $\mathcal{A}$ has an infinite computable chain $R$.

Proof. By the previous lemma, there is a fixed element $a_{0}$ with $\mu(x)=a_{0}$ for all $x \in R_{0}$. Let $R=\left\{x: a_{0} \leq x\right\}$. If $x \in R_{0}$, then $a_{0}=\mu(x) \leq x$, so $x \in R$. Hence $R_{0} \subseteq R$, so $R$ is infinite. As $a_{0} \in B_{\emptyset}, R$ is a chain by A7. Since $\mathcal{A}$ is computable, $R$ is also computable.

Let

$$
P=\left\{\sigma \in T:(\exists x)\left[x \in R \& x \in B_{\sigma}\right]\right\} .
$$

The next lemma gives us the desired contradiction.

Lemma 2.6. $P$ is a computable path through $T$.

Proof. For a chain of length 4 in $R$, the top element is in $B_{\emptyset}$, so $\emptyset$ is in $P$, by A5. For a chain of length 5 in $R$, the $4^{\text {th }}$ element is in $B_{\emptyset}$, so the top element must be 
in $B_{\sigma}$, where $\sigma$ has length 1 , by A3. In general, for a chain of length $n+4$ in $R$, the top element must be in $B_{\sigma}$, where $\sigma$ has length $n$. Thus, $P$ includes sequences of all finite lengths. By A3, all of these sequences are in $T$. If $\sigma, \tau \in P$, where we have $x<y$ in $R$ such that $x \in B_{\sigma}$ and $y \in B_{\tau}$, then, by definition, $y \in B_{\sigma}$, so, by A4, $\sigma$ and $\tau$ must be comparable. Therefore, $P$ is a path. Because $R$ is computable, $P$ is c.e., and because $P$ is a path, it follows easily that $P$ is computable.

We now prove the analogue of Theorem 2.1 for antichains, using a similar argument.

Theorem 2.7. There is a computably axiomatizable theory $K$ of partial orderings such that:

(1) $K$ has a computable model $\mathcal{D}$ with arbitrarily long finite antichains, and

(2) no computable model of $K$ has an infinite antichain.

Proof. Before giving the axioms for $K$, we describe the computable partial ordering $\mathcal{D}$ used for (1). The axioms for $K$ will then be a partial description of $\mathcal{D}$. Let the tree $T$ and the finite partial orderings $\left\{A_{n}\right\}_{n \in \omega}$ and $\left\{C_{n}\right\}_{n \in \omega}$ be as in the proof of Theorem 2.1. The model $\mathcal{D}$ will be the ordered sum of partial orderings $D_{q}$, for $q \in \mathbb{Q}$, where if $q<q^{\prime}$, then for all $x \in D_{q}$ and $y \in D_{q^{\prime}}$, we let $x<y$ in $\mathcal{D}$. We partition $\mathbb{Q}$ effectively into dense sets $\mathbb{Q}_{\sigma}$, for $\sigma \in T$. We let $D_{q} \cong C_{\sigma}$ if $q \in \mathbb{Q}_{\sigma}$.

We write $x \sim y$ if $x$ and $y$ belong to the same $D_{q}$. The relation $\sim$ is an equivalence relation which is definable in $\mathcal{D}$ by the formula saying that there exists $z$ incomparable with both $x$ and $y$. We say that $x \in B_{\sigma}$ if in the equivalence class of $x$ there is a copy of $A_{\sigma}$ containing an element $t<x$. This notion makes sense in any partial ordering in which the axiom A2 below is satisfied. Moreover, we can pass effectively from $\sigma$ to a formula saying that $x \in B_{\sigma}$. For $\mathcal{D}$, we have a strictly increasing computable function $b$ such that $b(n)$ is an upper bound on the size of the antichains in $C_{\sigma}$ for $\sigma$ of length at most $n$. We let $K$ be the theory generated by the following axioms.

A1. First, there are the axioms for partial orderings.

A2. There are axioms saying that the relation $x \sim y$ is an equivalence relation, where $x \sim y$ if there is some $z$ incomparable with both $x$ and $y$.

A3. There is an axiom saying that if $x \nsim y$, then $x<y$ or $y<x$.

A4. For each $n$, there is an axiom saying that for all $x$, if $x$ belongs to an antichain of size $\geq b(n)$, then some $y \sim x$ belongs to a chain of length $n+4$, where this chain is contained in the equivalence class of $x$.

A5. For each $\sigma \notin T$, there is an axiom saying that $B_{\sigma}$ is empty.

A6. For each $\sigma \in T$, there is an axiom saying that if $x<y, x \sim y$, and $x \in B_{\sigma}$, then $y \in B_{\sigma^{\wedge} 0}$ or $y \in B_{\sigma^{\wedge} 1}$.

A7. For all incompatible $\sigma, \tau \in T$, there is an axiom saying that $B_{\sigma} \cap B_{\tau}=\emptyset$.

A8. There is an axiom saying that for any chain of four elements in a single equivalence class, the greatest element is in $B_{\emptyset}$.

A9. There is an axiom saying that for any $x \in B_{\emptyset}$, there is a least $y \leq x$ such that $y \sim x$ and $y \in B_{\emptyset}$.

A10. Finally, there is an axiom saying that if $x \sim y$ and $x, y \in B_{\emptyset}$, then $x$ and $y$ are comparable. 
We can prove a lemma analogous to Lemma 2.2 characterizing the elements of $B_{\sigma}$ as the obvious ones. It follows easily that $\mathcal{D}$ is a model of $K$. Now, let $\mathcal{A}$ be a computable model of $K$. Suppose, for the sake of obtaining a contradiction, that $\mathcal{A}$ has an infinite antichain $R$. Say $r \in R$. By A3, the elements of $R$ must lie in a single equivalence class.

Lemma 2.8. The equivalence class of $r$ contains an infinite c.e. chain L, consisting exactly of the elements equivalent to $r$ and in $B_{\emptyset}$.

Proof. By A4, the equivalence class of $r$ contains arbitrarily large finite chains. For a chain of length $4+n$ in a single equivalence class, at least $n$ elements are in $B_{\emptyset}$. Thus, $L$ is infinite, where $L$ is the set of elements in $B_{\emptyset}$ and equivalent to $r$. By A10, $L$ is a chain. Also, $L$ is c.e., because the relation $\sim$ is c.e.

Let $P=\left\{\sigma \in T:(\exists x)\left[x \in L \& x \in B_{\sigma}\right]\right\}$. It follows as in Lemma 2.6 that $P$ is a computable path through $T$. This gives us the desired contradiction.

Above, we gave an example of a computably axiomatizable theory whose computable models have no infinite chains, although there are other models with infinite chains. We also gave an example of a theory whose computable models have no infinite antichains, although there are other models with infinite antichains. Next, we give an example of a theory whose computable models all have infinite chains, although there are other models with no infinite chains. We also give an example of a theory whose computable models all have infinite antichains, although there are other models with no infinite antichains.

Theorem 2.9. There is a complete theory $K$ of partial orderings such that $K$ has a computable model and

(1) every computable model of $K$ has infinite chains,

(2) $K$ has a model with no infinite chains.

Proof. The idea is the same as for a theory of equivalence structures which has computable models, and models with no infinite classes, but such that every computable model has infinitely many infinite classes (see Chapter 9 in [1]). Let $S$ be an infinite $\Delta_{2}^{0}$ set that does not contain the range of a computable limitwise monotonic function; i.e., there is no computable function $f(n, s)$ such that for all $n$, the function $f(n, s)$ is nondecreasing, with limit $m \in S$ such that $m \geq n$. Let $\mathcal{A}$ consist of disjoint maximal chains, one of size $n$ for each $n \in S$, and let $\bar{K}=T h(\mathcal{A})$. The model $\mathcal{A}$, which is the prime model of $K$, has no infinite chains. There are computable models of $K$, having infinitely many maximal infinite chains. Any computable model must have infinite chains; in fact, it must have infinitely many maximal infinite chains. If there were a computable model with only finitely many, then we could produce a computable function $f(n, s)$ of the kind that does not exist.

Theorem 2.10. There is a complete theory $K$ of partial orderings such that $K$ has a computable model and

(1) every computable model of $K$ has infinite antichains,

(2) $K$ has a model with no infinite antichains.

Proof. As in the previous theorem, we let $S$ be an infinite $\Delta_{2}^{0}$ set does not contain the range of any computable limitwise monotonic function. We may suppose that 
$0 \notin S$. We partition $\mathbb{Q}$ effectively into dense sets $\mathbb{Q}_{n}$, for $n \in S$. Let $K$ be the theory of the structure $\mathcal{A}$ that results from replacing $q$, for $q \in \mathbb{Q}_{n}$, by an antichain of size $n$. There are computable models of $K$ having densely many infinite antichains. A model which does not have densely many infinite antichains cannot be computable. If $x<y$ in a computable model and there are no infinite antichains between $x$ and $y$, then we could again produce a computable function $f(n, s)$ of the kind that does not exist.

\section{Uniformity FOR $\Sigma_{2}^{0}$ CHAins AND $\Sigma_{2}^{0}$ ANTICHAINS}

In this section, we consider computable partial orderings $\mathcal{A}$ with the feature that for every isomorphic copy $\mathcal{B}$, there is an infinite chain or antichain that is $\Sigma_{2}^{0}$ relative to $\mathcal{B}$, where $\mathcal{B}$ is identified with its atomic diagram. We note that an infinite set that is $\Sigma_{2}^{0}$ relative to $\mathcal{B}$ has an infinite subset that is $\Delta_{2}^{0}$ relative to $\mathcal{B}$. We let $\Delta_{2}^{0}(\mathcal{B})$ denote the class of sets $\Delta_{2}^{0}$ relative to $\mathcal{B}$. Our result is a uniform dichotomy-either there is always an infinite chain that is $\Delta_{2}^{0}(\mathcal{B})$, or else there is always an infinite antichain that is $\Delta_{2}^{0}(\mathcal{B})$.

Theorem 3.1. Let $\mathcal{A}$ be a computable partial ordering such that for each $\mathcal{B} \cong \mathcal{A}$ there is an infinite chain or antichain that is $\Delta_{2}^{0}(\mathcal{B})$. Then either for all $\mathcal{B} \cong \mathcal{A}$ there is an infinite chain that is $\Delta_{2}^{0}(\mathcal{B})$, or else for all $\mathcal{B} \cong \mathcal{A}$ there is an infinite antichain that is $\Delta_{2}^{0}(\mathcal{B})$. Moreover, there is a finite tuple $\bar{c}$ in $\mathcal{A}$ such that for $(\mathcal{B}, \bar{d}) \cong(\mathcal{A}, \bar{c})$, the infinite chain (or antichain) is uniformly $\Delta_{2}^{0}(\mathcal{B}, \bar{d})$.

There are several results on objects "relatively intrinsically" in some complexity class $\Gamma$. The idea behind all of these results is that if for all isomorphic copies $\mathcal{B}$ of the given $\mathcal{A}$, something "intrinsic" to the structure has complexity $\Gamma$ relative to $\mathcal{B}$, then using forcing, we can extract syntactical conditions that account for the bounds. Some results of this kind are gathered together in Chapter 10 of [1].

We could prove Theorem 3.1 directly in this way. As in the other results, we build a "generic" copy $\mathcal{A}^{*}$ of $\mathcal{A}$. The forcing conditions are finite partial $1-1$ functions, which we think of as possible partial isomorphisms from $\mathcal{A}^{*}$ to $\mathcal{A}$. Since $\mathcal{A}^{*} \cong \mathcal{A}$, there is an infinite chain or antichain $C$ that is $\Delta_{2}^{0}\left(\mathcal{A}^{*}\right)$. Say that $C$ is a chain, and let $e$ be an index for $C$ as a set that is $\Delta_{2}^{0}\left(\mathcal{A}^{*}\right)$. It follows (from a lemma on Truth and Forcing) that some forcing condition $p$ must force the statement that the $\Delta_{2}^{0}\left(\mathcal{A}^{*}\right)$ set with index $e$ is an infinite chain. Let $\bar{c}$ be the range of $p$. If $(\mathcal{B}, \bar{d}) \cong(\mathcal{A}, \bar{c})$, then, thinking about forcing for building a generic copy of $\mathcal{B}$, we could produce an infinite chain that is $\Delta_{2}^{0}(\mathcal{B})$.

Instead of filling in this outline, doing the forcing construction from scratch, we apply an earlier result, Theorem 2.6 from [2], which was also obtained by forcing. The setting from [2] is as follows. Let $\mathcal{A}$ be a computable structure for the language $L$, and let $\psi$ be a sentence involving a new relation symbol $R$. The theorem gives syntactical conditions guaranteeing that for all $\mathcal{B} \cong \mathcal{A}$, there exists $R$ such that $(\mathcal{B}, R) \models \psi$ and $R$ is $\Delta_{\alpha}^{0}(\mathcal{B})$. The sentence $\psi$ must have a special form.

Definition 3.2. We say that a formula $\psi$ is $\Pi_{\alpha+1}$ given that $R$ is $\Delta_{\alpha}$, and write $\Pi_{\alpha+1} \mid\left(R \in \Delta_{\alpha}\right)$, if $\psi$ has the form

$$
\mathbb{A}_{i}\left(\forall \bar{u}_{i}\right) \underset{j}{\mathbb{\bigvee}}\left(\exists \bar{v}_{i, j}\right)\left[\varphi_{i, j}\left(\bar{u}_{i}, \bar{v}_{i, j}\right) \wedge \rho_{i, j}\left(\bar{u}_{i}, \bar{v}_{i, j}\right)\right],
$$


where for each $i, j$, the formula $\varphi_{i, j}$ is computable $\Pi_{\gamma}$ in the language $L$, for some $\gamma<\alpha$, and $\rho_{i, j}$ is an $R$-formula, where an $R$-formula is a finite conjunction of atomic formulas and negations of atomic formulas using the predicate $R$.

The syntactical conditions are like those for relative $\Delta_{\alpha}^{0}$-categoricity in that they involve a family of formulas with a fixed tuple of parameters. We say that a tuple of constants $\bar{c}$ is appropriate for a tuple of variables $\bar{u}$ if they are of the same length.

Definition 3.3. Let $\psi=\mathbb{A}_{i}\left(\forall \bar{u}_{i}\right) \mathbb{W}_{j}\left(\exists \bar{v}_{i, j}\right)\left[\varphi_{i, j}\left(\bar{u}_{i}, \bar{v}_{i, j}\right) \wedge \rho_{i, j}\left(\bar{u}_{i}, \bar{v}_{i, j}\right)\right]$ be as above. An expansion family for $\psi$ on $\mathcal{A}$ is a family $\mathcal{S}$ of consistent $R$-sentences with constants from $\mathcal{A}$ such that:

(1) $\mathcal{S} \neq \emptyset$,

(2) if $\sigma \in \mathcal{S}$ and $a \in \mathcal{A}$, then there exists $\tau \in \mathcal{S}$ such that $\tau \vdash \sigma$ and either $\tau \vdash R a$ or $\tau \vdash \neg R a$,

(3) for each $\sigma \in \mathcal{S}, i \in \omega$, and $\bar{c}$ in $\mathcal{A}$ (appropriate for $\bar{u}_{i}$ ), there exist $\tau \in \mathcal{S}$, $j \in \omega$, and $\bar{d}$ in $\mathcal{A}$ (appropriate for $\bar{v}_{i, j}$ ) such that $\tau \vdash \sigma, \tau \vdash \rho_{i, j}$, and $\mathcal{A} \models \varphi_{i, j}(\bar{c}, \bar{d})$.

We say that $\mathcal{S}$ is a formally $\Sigma_{\alpha}^{0}$ expansion family for $\psi$ on $\mathcal{A}$ if for some finite sequence $\bar{c}$ in $\mathcal{A}$, there is a computable function assigning to each $R$-formula $\sigma(\bar{x})$ a computable $\Sigma_{\alpha}$ formula $\alpha_{\sigma}(\bar{c}, \bar{x})$, in the language $L$, such that for $\bar{a}$ in $\mathcal{A}, \sigma(\bar{a}) \in \mathcal{S}$ iff $\mathcal{A} \models \alpha_{\sigma}(\bar{c}, \bar{a})$.

The result that we need to prove Theorem 3.1, Theorem 2.6 from [2], is stated below.

Theorem. Let $\mathcal{A}$ be a computable structure, and let $\psi$ be a sentence involving a new relation symbol $R$, where $\psi$ is computable $\Pi_{\alpha+1} \mid\left(R \in \Delta_{\alpha}\right)$. Then the following are equivalent:

(1) For all $\mathcal{B} \cong \mathcal{A}$, there exists $R$ such that $(\mathcal{B}, R) \models \psi$ and $R$ is $\Delta_{\alpha}^{0}(\mathcal{B})$.

(2) There is a formally $\Sigma_{\alpha}^{0}$ expansion family for $\psi$ on $\mathcal{A}$.

To make the notion of $\Sigma_{\alpha}^{0}$ expansion family more concrete, we sketch the easy direction of the proof.

Sketch of proof that $(2) \Rightarrow(1)$. Let $\mathcal{S}$ be a formally $\Sigma_{\alpha}^{0}$ expansion family for $\psi$ on $\mathcal{A}$, witnessed by the computable function $\alpha$ taking the $R$-formula $\sigma$ to the computable $\Sigma_{\alpha} L$-formula $\alpha_{\sigma}(\bar{c}, \bar{x})$ with parameters $\bar{c}$. Suppose $(\mathcal{B}, \bar{d}) \cong(\mathcal{A}, \bar{c})$. We get a formally $\Sigma_{\alpha}^{0}$ expansion family $\mathcal{S}^{\prime}$ for $\psi$ on $\mathcal{B}$, witnessed by the computable function $\alpha^{\prime}$ that takes the $R$-formula $\sigma(\bar{x})$ to $\alpha_{\sigma}(\bar{d}, \bar{x})$. Using $\alpha^{\prime}$, and looking at the given sentence

$$
\psi=\mathbb{A}_{i}\left(\forall \bar{u}_{i}\right) \underset{j}{\mathbb{V}}\left(\exists \bar{v}_{i, j}\right)\left[\varphi_{i, j}\left(\bar{u}_{i}, \bar{v}_{i, j}\right) \wedge \rho_{i, j}\left(\bar{u}_{i}, \bar{v}_{i, j}\right)\right]
$$

we can produce $R$ such that $(\mathcal{B}, R) \models \psi$ and $R$ is $\Delta_{\alpha}^{0}(\mathcal{B})$. Given a pair $i$ and $\bar{u}_{i}$, we look for $j$ and $\bar{v}_{i, j}$ such that $\mathcal{B}=\varphi_{i, j}\left(\bar{u}_{i}, \bar{v}_{i, j}\right)$ and for some $R$-sentence $\rho(\bar{b})$, $\rho \vdash \rho_{i, j}\left(\bar{u}_{i}, \bar{v}_{i, j}\right)$ and $\mathcal{B} \models \alpha_{\rho}^{\prime}(\bar{d}, \bar{b})$. We determine enough of $R$ to make $\rho(\bar{b})$ true. Having decided $\rho(\bar{b})$, we choose another pair $i$ and $\bar{u}_{i}$. We look for $j$ and $\bar{v}_{i, j}$ such that $\mathcal{B} \models \varphi_{i, j}\left(\bar{u}_{i}, \bar{v}_{i}\right)$ and for some $R$-sentence $\rho^{\prime}\left(\bar{b}^{\prime}\right), \rho^{\prime}\left(\bar{b}^{\prime}\right) \vdash \rho(\bar{b}) \& \rho_{i, j}\left(\bar{u}_{i}, \bar{v}_{i, j}\right)$. Continuing in this way, we arrive at the desired $R$. 
For our application, $\alpha=2$. Our sentence $\psi$ says that $R$ is infinite, and that it is either a chain or an antichain. It is easy to put $\psi$ in the required form $\Pi_{3} \mid\left(R \in \Delta_{2}\right)$. There is an infinite family of conjuncts, which, taken all together, say that $R$ is infinite. For each $n$, we have a conjunct $\left(\forall u_{1}, \ldots, u_{n}\right)(\exists v)\left[R v \& \wedge_{i=1}^{n} v \neq u_{i}\right]$.

The sentence $\psi$ has a final conjunct saying that $R$ is either a chain or an antichain. The sentence

$$
\left(\forall u_{1}\right)\left(\forall u_{2}\right)\left[u_{1}<u_{2} \vee u_{2}<u_{1} \vee u_{1}=u_{2} \vee \neg R u_{1} \vee \neg R u_{2}\right]
$$

says that $R$ is a chain (if both $u_{1}$ and $u_{2}$ are in $R$, then they are comparable). Each disjunct here is either a quantifier-free $L$-formula or an $R$-formula. The sentence

$$
\left(\forall u_{3}\right)\left(\forall u_{4}\right)\left[\left(\neg u_{3}<u_{4} \& \neg u_{4}<u_{3}\right) \vee \neg R u_{3} \vee \neg R u_{4} \vee u_{3}=u_{4}\right]
$$

says that $R$ is an antichain (if $u_{3}$ and $u_{4}$ are distinct elements of $R$, then they are incomparable). Again, each disjunct here is either a quantifier-free $L$-formula or an $R$-formula. The sentence we want for the last conjunct is $\left(\forall u_{1}, u_{2}, u_{3}, u_{4}\right)\left[\gamma_{1} \vee \gamma_{2}\right]$, where the two sentences above are $\left(\forall u_{1}\right)\left(\forall u_{2}\right) \gamma_{1}$ and $(\forall u)\left(\forall u_{4}\right) \gamma_{2}$.

We are in a position to apply the theorem from [2], stated above. We get a formally $\Sigma_{2}^{0}$ expansion family $\mathcal{S}$. Let $\alpha$ be the computable function taking $R$ formulas $\sigma$ to computable $\Sigma_{2}^{0} L$-formulas $\alpha_{\sigma}(\bar{c}, \bar{u})$, with parameters $\bar{c}$, such that $\sigma(\bar{a}) \in \mathcal{S}$ iff $\mathcal{A}=\alpha_{\sigma}(\bar{c}, \bar{a})$. Clearly, there must exist distinct $a_{1}, a_{2}$ in $\mathcal{A}$ such that for some $\sigma \in \mathcal{S}, \sigma \vdash R a_{1} \& R a_{2}$. We form a new $\Sigma_{2}^{0}$ expansion family $\mathcal{S}^{\prime}$, witnessed by a function $\alpha^{\prime}$. We let $\mathcal{S}^{\prime}$ consist of those $\tau \in \mathcal{S}$ such that $\tau \vdash R a_{1} \& R a_{2}$. Given an $R$-formula $\sigma$, we let $\alpha_{\sigma^{\prime}}^{\prime}\left(\bar{c}^{\prime}, \bar{u}\right)$ be $\alpha_{\sigma^{\prime}}\left(\bar{c}, a_{1}, a_{2}, \bar{u}\right)$. We have

$$
\sigma^{\prime}(\bar{a}) \in \mathcal{S}^{\prime} \text { iff } \mathcal{A} \models \alpha_{\sigma^{\prime}}^{\prime}\left(\bar{c}^{\prime}, \bar{a}\right) .
$$

Suppose $(\mathcal{B}, \bar{d}) \cong(\mathcal{A}, \bar{c})$. If $\left(\mathcal{B}, \bar{d}, b_{1}, b_{2}\right) \cong\left(\mathcal{A}, \bar{c}, a_{1}, a_{2}\right)$, then $b_{1}, b_{2}$ are comparable just in case $a_{1}, a_{2}$ are. Using $\mathcal{S}^{\prime}$ and the formulas $\alpha_{\sigma^{\prime}}^{\prime}\left(\bar{d}, b_{1}, b_{2}\right)$, we obtain an infinite set $R$ that is $\Delta_{2}^{0}(\mathcal{B})$ and includes $b_{1}, b_{2}$. Now, $R$ is a chain if $a_{1}, a_{2}$ are comparable and an antichain otherwise, and this fact is independent of $\mathcal{B}$. Thus, we have the desired uniform dichotomy. This completes the proof of Theorem 3.1.

There are nontrivial examples of computable partial orderings $\mathcal{P}$ such that every copy $\mathcal{B}$ of $\mathcal{P}$ has an infinite chain or antichain that is $\Delta_{2}^{0}(\mathcal{B})$. In fact, it is shown in [4] that there is a computable partial ordering $\mathcal{P}$ such that $\mathcal{P}$ has no infinite $\Pi_{1}^{0}$ chains or antichains, and every copy $\mathcal{B}$ of $P$ has an infinite chain that is $\Delta_{2}^{0}(\mathcal{B})$ and also an infinite antichain that is $\Delta_{2}^{0}(\mathcal{B})$. The key to ensuring the latter property is to make $\mathcal{P}$ weakly stable, in the sense that for every $a$ of its field $P$, either all but finitely many elements of $P$ lie below $a$, or all but finitely many elements of $P$ lie above $P$, or all but finitely many elements of $P$ are incomparable with $a$. It is easily seen that if $\mathcal{B}$ is weakly stable and has no infinite $\mathcal{B}$-computable chains or antichains, then $\mathcal{B}$ has an infinite $\Delta_{2}^{0}(\mathcal{B})$ chain and also an infinite $\Delta_{2}^{0}(\mathcal{B})$ antichain. The main step is to construct a computable weakly stable partial ordering with no infinite $\Pi_{1}^{0}$ chains or antichains.

\section{Problems}

The first problem is related to Theorem 2.9. In a sense, it asks whether the theory $K$ in that result can be made computably axiomatizable. 
Problem 1. Is there a computably axiomatizable theory of partial orderings such that some model has arbitrarily large finite chains and no infinite chain, there is a computable model, and every computable model has an infinite chain?

The next problem is the analogous question for antichains and is related to Theorem 2.10.

Problem 2. Is there a computably axiomatizable theory of partial orderings such that some model has arbitrarily large finite antichains and no infinite antichain, there is a computable model, and every computable model has an infinite antichain?

The next question asks whether the analogue of Theorem 1.2 holds for antichains.

Problem 3. Is there a computable partial ordering $\mathcal{A}$ with an infinite antichain but not one that is $d-\Pi_{1}^{1}$ ?

In connection with this last problem, we have shown that there is no effective procedure which, given an index of a computable partial ordering $\mathcal{A}$ with an infinite antichain, produces two indices $i_{1}, i_{2}$ of $d$ - $\Pi_{1}^{1}$ sets, such that either $i_{1}$ or $i_{2}$ is an index of the "leftmost" infinite antichain $C$ of $\mathcal{A}$. It seems plausible that this result can be extended to cover any finite set of $d$ - $\Pi_{1}^{1}$ indices, but we have no idea how to handle all indices simultaneously, nor how to deal with other potential antichains.

\section{REFERENCES}

[1] C. J. Ash and J. F. Knight, Computable Structures and the Hyperarithmetical Hierarchy, Elsevier, Amsterdam, 2000.

[2] C. J. Ash and J. F. Knight, Relatively recursive expansions, Fundamenta Mathematicae 140 (1992), pp. 137-155.

[3] S. Binns, B. Kjos-Hanssen, M. Lerman, J. H. Schmerl, and R. Solomon, Self-embeddings of computable trees, to appear in the Notre Dame Journal of Formal Logic.

[4] C. Jockusch, Jr., B. Kastermans, S. Lempp, M. Lerman, and R. Solomon, Stable and weakly stable partial orderings, in preparation.

[5] E. Herrmann, Infinite chains and antichains in computable partial orderings, Journal of Symbolic Logic 66 (2001), pp. 923-934.

[6] C. G. Jockusch, Jr., Ramsey's theorem and recursion theory, Journal of Symbolic Logic 37 (1972), pp. 268-280.

[7] H. Rogers, Jr., Theory of Recursive Functions and Effective Computability, McGraw-Hill, New York, 1967.

Department of Mathematics, George Washington University, Washington, DC 20052, HARIZANV@GWU.EDU

Department of Mathematics, University of Illinois at Urbana-Champaign, 1409 W. Green St., Urbana, IL 61801, Jockusch@math.uiuc.edu

Department of Mathematics, University of Notre Dame, Notre Dame, IN 46556 , KNIGHT.1@ND.EDU 